\title{
Циркулирующий гликопротеин 130 у пациентов с различной степеныю выраженности симптомов хронической сердечной недостаточности
}

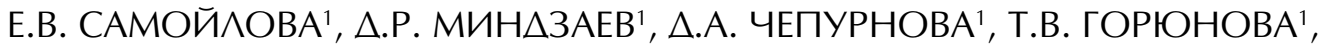 \\ И.В. ЖИРОВ ${ }^{1,2}$, С.Н. НАСОНОВА ${ }^{1}$, А.А. KOPOТАЕВА ${ }^{1}$, С.Н. ТЕРЕШЕНКО ${ }^{1,2}$ \\ 'ФГБУ «Национальный медицинский исследовательский центр кардиологии» Минздрава России, Москва, Россия

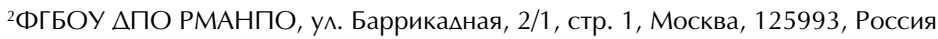

\begin{abstract}
Аннотация. Растворимая форма гликопротеина 130 (sgp130) является ингибитором транссигнального пути передачи сигнала И^-6 (интерлейкина-6). Препятствуя проведению сигнала, sgp130 может оказывать влияние на развитие И^-6-ассоциированных патологий. Цель исследования. Оценить циркулируюшие уровни sgp130 у пациентов с различной этиологией и степенью выраженности симптомов хронической сердечной недостаточности (ХCH). Материал и методы. В исследование были включены 61 пациент с ХСН и 14 здоровых Аоноров. Причиной сердечной недостаточности у 29 пациентов была ишемическая болезнь сердца (ИБС), у 19 пациентов артериальная гипертензия (АГ) и у 13 пациентов диматационная кардиомиопатия (АКМП). В зависимости от выраженности симптомов ХСН пациенты были отнесены к II (n = 20) и III (n = 41) функциональным классам (ФК). Результаты. У пациентов с ХСН циркулируюшие уровни sgр130 значительно выше, чем у здоровых доноров. В группах пациентов, сформированных в соответствии с этиологией XСН, концентрации sgp130 достоверно не различались. В группах пациентов, сформированных в зависимости от выраженности симптомов заболевания, уровни sgp130 были значительно выше у пациентов III ФК по сравнению с пациентами II ФК. Аогистический регрессионный анализ с включением в модель возраста, пола, показателя фракции выброса левого желудочка (ФВ АЖ), АГ, ИБС, сахарного Аиабета, диастолической $и$ ифункции, статинов, NT-proBNP (N-терминальный пропепти мозгового натрийуретического гормона), креатинина подтверАил статистически значимую связь между уровнями sgp130 и выраженностью симптомов заболевания (ОШ = 1,019 ( $\Delta$ И 1,006-1,0031), p = 0.003). Между уровнями sgp130 и NT-pro-BNP у всех исследуемых пациентов с ХСН выявлена положительная корреляционная связь. Выводы. Циркулируюшие уровни sgp130 повышаются при прогрессировании ХСН вне зависимости от этиологии заболевания.
\end{abstract}

Киючевые слова: растворимый гликопротеин 130, хроническая сердечная недостаточность, выраженность симптомов заболевания, этиология.

\section{Circulating glycoprotein 130 in patients with chronic heart failure of varying symptom degrees}

\author{
E.V. SAMOILOVA ${ }^{1}$, D.R. MINDZAEV ${ }^{1}$, D.A. CHEPURNOVA ${ }^{1}$, T.V. GORUNOVA ${ }^{1}$ \\ I.V. ZHIROV ${ }^{1,2}$, S.N. NASONOVA ${ }^{1}$, A.A. KOROTAEVA ${ }^{1}$, S.N. TERESCHENKO ${ }^{1,2}$
}

'Federal State budget organization «National medical research center of cardiology» of the Ministry of healthcare of the Russian Federation, Moscow, Russia; ${ }^{2}$ FSBEl FPE Russian Medical Academy of Continuous Professional Education, Moscow, Russian Federation

\begin{abstract}
IL-6 trans-signaling pathway. Sgp130 can affect the development of the IL-6-associated pathologies by preventing the IL-6 signal transduction. Aim. We examined circulating levels of sgp130 in patients with chronic heart failure (CHF) of different etiologies and varying severity of symptoms. Material and methods. The study included 61 patients with $\mathrm{CHF}$ and 14 healthy donors. The cause of heart failure was coronary heart disease (CHD) in 29 patients, arterial hypertension (AH) in 19 patients and dilated cardiomyopathy (DCMP) in 13 patients. The patients were assigned to II $(n=20)$ and III $(n=41)$ functional classes $(F C)$ depending on the severity of the CHF symptoms. Results. The circulating levels in patients with CHF were significantly higher than in healthy donors. The levels of sgp130 did not differ significantly in the groups of patients formed according to CHF etiology. Sgp130 levels were significantly higher in patients with III FC compared with those in patients with II FC. The multivariate linear regression analysis including sex, age, left ventricular ejection fraction, diabetes status, statins, arterial hypertension, coronary artery disease, NT-proBNP has revealed the association between sgp130 levels and disease severity: $\mathrm{OR}=1.019(95 \% \mathrm{Cl}: 1.006-10031), \mathrm{p}=0.003$. The positive correlation between sgp130 and NT-proBNP levels $(r=0.324, p=0.011)$ was established in all patients. Conclusion. The circulating levels of sgp130 increase with the progression of $\mathrm{CHF}$ regardless of the disease etiology.
\end{abstract}

Keywords: soluble glycoprotein 130, chronic heart failure, disease severity, etiology. 
Сведения об авторах:

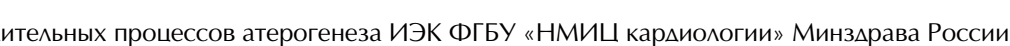
erihter@mail.ru (автор, ответственный за переписку)

А.Р. Миннзаев - аспирант отАела заболеваний миокарда и сердечной немостатоности ИКК иМ. А.А. Мясникова ФГБУ «НМИЦ карАиологии Минзарава России

А.А. Чепурнова - М. н. с. Ааб. биохимии воспалитемьных процессов атерогенеза ИЭК ФГБУ «НМИЦ кардиологии» Минздрава России

т.В. Горюнова - врач-карАиолог 8-го карАиологического отАеления ИКК им. А.А. Мясникова ФГБУ «НМИЦ карАиологиия Минздрава России С.Н. Насонова - к. М. н., с. н. с. отАела заболеваний миокарда и сердечной недостаточности ИКК им. А.А. Мясникова ФГБУ «НМИЦ карАиомогии» Минздрава России

и.в. Жиров - А. м. н., ведушии научныи сотрудник отдела заболевании миокарда и сердечной недостатои оси

ФГБУ «НМИЦ карАиологии» Минздрава России, профессор кафедры карАиологии ФГБОУ АПО РМАНПО

А.А. Коротаева - А. б. н., рук. Ааб. биохимии воспалительных процессов атерогенеза ИЭК ФГБУ «НМИЦ карАиологии» Минздрава России.

3-я Черепковская, 15а, Москва, 12155

С.н. Тережеко,

ности ИКК им. АА. М

\section{Введение}

Хроническая сердечная недостаточность (ХСН) является одной из основных проблем общественного здоровья и здравоохранения. ХСН характеризуется клиническими симптомами, связанными с нарушением функции сердца. В оценке ХСН важную роль играют биомаркеры, такие как мозговой натрийуретический пептид (BNP) и его предшественник, $\mathrm{N}$-терминальный пропептид мозгового натрийуретического гормона (NT-proBNP) [1]. Однако, несмотря на свою значимость, данные биомаркеры имеют ограничения в диагностике ХСН[2]. В этой связи в клинической практике необходимь новые биомаркеры, которые будут способствовать выявлению субклинических стадий ХCH, выбору и контролю лечения, мониторингу лечения и стратификации рисков.

Как известно, в патогенез ХCH вовлечено воспаление. Интерлейкин-6 (ИЛ-6) является одним из наиболее важных медиаторов воспаления и рассматривается в качестве прогностического предиктора у пациентов с сердечной недостаточностью [3-6]. Провоспалительные эффекты ИЛ-6 реализуются транссигнальным путем [7]. Естественным ингибитором транссигнального пути, циркулирующим в плазме крови, является растворимый гликопротеин gp130 (sgp 130), представляющий собой растворимую форму одной из субъединиц рецептора ИЛ-6 [8]. Предотвращая проведение сигнала, sgp130 может оказывать влияние на развитие ИЛ-6-ассоциированных патологий. Показано, что уровень sgp 130 меняется в зависимости от интенсивности воспалительного процесса [9]. Следовательно, можно предположить, что концентрация sgp 130 меняется у пашиентов с XCH, отражая степень тяжести заболевания.

В настоящем исследовании оценивали циркулирующие уровни sgp 130 у пашиентов с различной этиологией и функциональными классами ХСН.

\section{Методы}

Исследование включало 61 пациента (56 мужчин и 5 женщин) с острой декомпенсацией ХСН и 14 здоровых добровольцев. Все участники исследования подписали иней Нью-Йоркской кардиологической ассоииаии (NYHA), пациенты с ХСН были отнесены к II ( $\mathrm{n}=20)$ и ІІІ ( $\mathrm{n}=41)$ ФК (табл. 1).

Основными заболеваниями, явившимися причиной сердечной недостаточности у данных пациентов, был ИБС $(\mathrm{n}=29), А Г(~(n=19)$ и ДКМП $(\mathrm{n}=13)$. У 15\% пациентов II ФК $(\mathrm{n}=3)$ и у $20 \%$ пациентов III ФК $(\mathrm{n}=8$ среди сопутствующих заболеваний имелся СД. Критериями исключения являлись острые и хронические воспалиельные заболевания, требовавшие проведения специфической противовоспалительной терапии, потенциально влияющей на изучаемые параметры, выраженные нару шения функции печени и/или почек, наличие злокачественных новообразований, а также наличие обструкции выносящего тракта левого желудочка.

\section{Таблица 1. Характеристики пациентов}

\begin{tabular}{|c|c|c|c|}
\hline Параметр & $\| \Phi K(n=20)$ & III $\Phi K(n=41)$ & p \\
\hline Возраст & $\begin{array}{c}50,5 \\
(42,75-58,25)\end{array}$ & $\begin{array}{c}62 \\
(53-67)\end{array}$ & 0,003 \\
\hline $\begin{array}{l}\text { Мужчины/женшины, } \\
\text { абс. (\%) }\end{array}$ & 95/5 & $85 / 15$ & 0,409 \\
\hline ФВ ^Ж, абс. (\%) & $\begin{array}{c}27,5 \\
(25-31,75)\end{array}$ & $\begin{array}{c}30 \\
(23,5-35)\end{array}$ & 0,595 \\
\hline $\begin{array}{l}\text { Аиастолическая } \\
\text { Аисфункция, абс. (\%) }\end{array}$ & 75 & 80 & 0,741 \\
\hline Статины, абс. (\%) & 50 & 54 & 1,00 \\
\hline АГ, абс. (\%) & 60 & 83 & 0,064 \\
\hline ИБС, абс. (\%) & 25 & 61 & 0,013 \\
\hline NT- proBNP (пr/MN) & $\begin{array}{c}395,5 \\
(224,5-825)\end{array}$ & $\begin{array}{c}793 \\
(408,5-1746,5)\end{array}$ & 0,009 \\
\hline Креатинин (мкмоль/А) & $\begin{array}{c}88 \\
(74-130)\end{array}$ & $\begin{array}{c}101 \\
(72,5-112,25)\end{array}$ & 0,432 \\
\hline Sgp130 (Hr/MN) & $\begin{array}{c}333 \\
(309-359)\end{array}$ & $\begin{array}{c}415 \\
(355-469)\end{array}$ & 0,001 \\
\hline CA, абс. (\%) & $3(15)$ & $8(20)$ & 0,350 \\
\hline
\end{tabular}

Примечание. Аанные премставеены как мемиана $(25$ прочентим

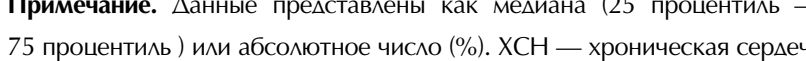

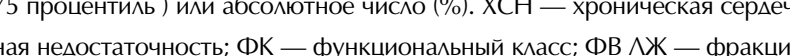
выброса левого желудочка; АГ - артериальная гипертензия; ИБС ишемическая болезнь сер

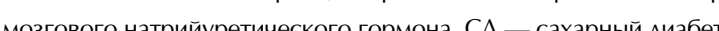

Биохимические методы

Венозную кровь отбирали в пробирки с ЭДТА. Плазму для исследований получали путем центрифугирования при $1000 \mathrm{~g}$ в течение 20 мин. Образцы плазмы крови хранили при $-70{ }^{\circ} \mathrm{C}$ С до исследования.

Концентрации sgp 130 в плазме крови определяли иммуноферментным методом (ELISA) с использованием наборо реактивов фирмы R\&D Systems, USA. Чувствительность метода составляла $80 \mathrm{pg} / \mathrm{ml}$. Определение концентрации NTproBNP в крови проводили электрохемилюминесцентным способом на анализаторе Elecsys 2010 (Roche, Швейцария) с использованием соответствующих коммерческих наборов Диапазон измерений NT-proBNP: 5-35 000 пг/мл.

\section{Статистические методы}

Статистический анализ полученных данных проводили с использованием пакета статистических программ SPSS Statistics 19. Результаты представлены в виде медианы с указанием интерквартильного интервала (25-й процентиль; 75-й процентиль) для параметров, имеющих распределение, отлич ное от нормального. При сравнении группп по количественному признаку использовались непараметрические (критерии Манна - Уитни и Краскела - Уоллиса) методы. При оценке достоверности различий частоты качественных показателей применяли двухсторонний точный критерий Фишера. Для выявления зависимости между показателями применяли коэффициент ранговой корреляции Спирмена и логистический результаты при уровне достоверности $\mathrm{p}<0,05$.

\section{Результать}

Сравнение циркулирующих уровней sgp 130 у всех исследуемых пациентов с ХСН и здоровых доноров показало, что у пациентов с XСН концентрации sgpl30 были значигельно выше (374 (328,5-438,5) нг/мл, чем у здоровых доноров 326,52 (296,56-360,82) нг/мл, $\mathrm{p}=0.007$ ) (рис. 1 ).

В соответствии с причинами развития сердечнои недостаточности пашиенты были поделены на три груп-

\section{Рисунок 1. Уровни sgp130 у пациентов с ХСН и зАоровых} Аоноро
700

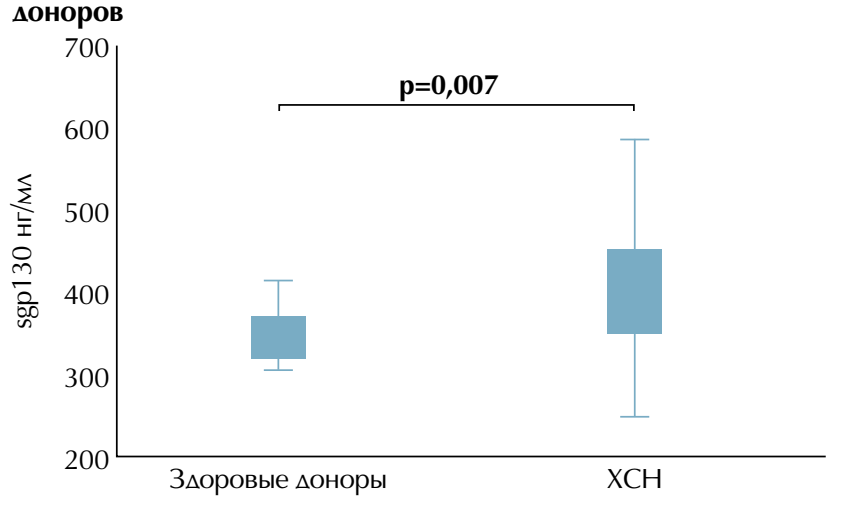

Максимаььные и минимахьные значения, интерквартимьный размах и медианы концентрации sgp 130; p - значение приведено Аяя крите рия Манна - Уитни
Рисунок 2. Уровни sgp130 у пашиентов в зависимости $\mathrm{XCH}$

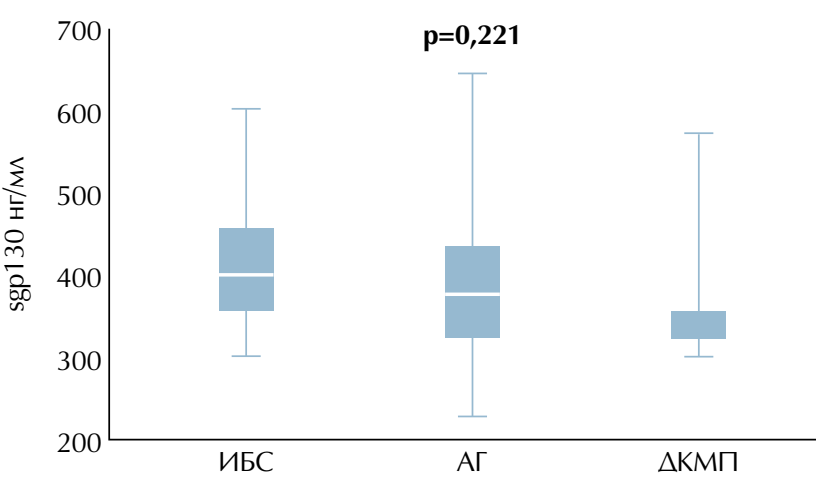

Максимахьные и минимахьные значения, интерквартимьныи размах

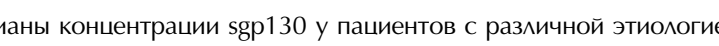
$\mathrm{XCH} ; \mathrm{p}$ - значение приведено цмя критерия Крускама - Уолисса.

пы: группа ИБС, группа АГ и группа ДКМП. Результать 质уемых групп, выполненных с помощь критерия Краскела - Уоллиса, не выявили достоверных различий уровней sgp130 между группами (рис. 2).

Концентрации NT-proBNP в группах с различной этиологией ХСН также значительно не различалис между собой. В группе ИБС его значение составило 822 (318-1615) пг/мл, в группе АГ- $703(353-1496)$ пг/мл, в группе ДКМП - $422(184,5-804)$ пг/мл.

Сравнительный анализ sgp130 и NT-proBNP в зависимости от выраженности симптомов заболевания показал, что уровни sgp130 и NT-proBNP были значительно выше у пациентов III ФК по сравнению с пациентами II ФК (рис. 3).

Логистический регрессионный анализ с включением в модель возраста, пола, показателя ФВ ЛЖ, АГ, ИБС, СД, диастолической дисфункции, статинов, NT-proBNP, креатинина подтвердил статистически значимую связ между уровнями sgp130 и выраженностью симптомов заболевания (ОШ =1,019 (ДИ 1,006-1,0031)), $\mathrm{p}=0,003$ ).

Корреляционный анализ выявил положительную связь между уровнями sgp130 и NT-proBNP у всех исследованных пациентов с ХCH (рис. 4.)

\section{Обсужиение}

В данном исследовании наблюдалось повышение уровней sgp130 у пациентов с ХCH. В литературе есть даннье об изменении уровней sgp130 при различных патологиях Показано, что концентрации sgp130 положительно коррелировали с артериальным давлением у пациентов с АГ [10] Показано, что уровни sgp 130 снижаются у пациентов со стабильной ИБС при прогрессировании атеросклероза [11] и во время течения острого инфаркта миокарда [12]. Hirotac c соавт. наблюдали увеличение уровней sgp 130 у пашиенто с застойной хронической недостаточностью [13].

Таким образом, циркулирующие уровни sgp 130 измения организма.

Сердечная недостаточность может быть проявление Срактически всех болезней серлие. Лечение серлечной не- 


\section{Рисунок 3. Уровни sgp130 и NT-proBNP}

У пашиентов с XCH II и III ФК

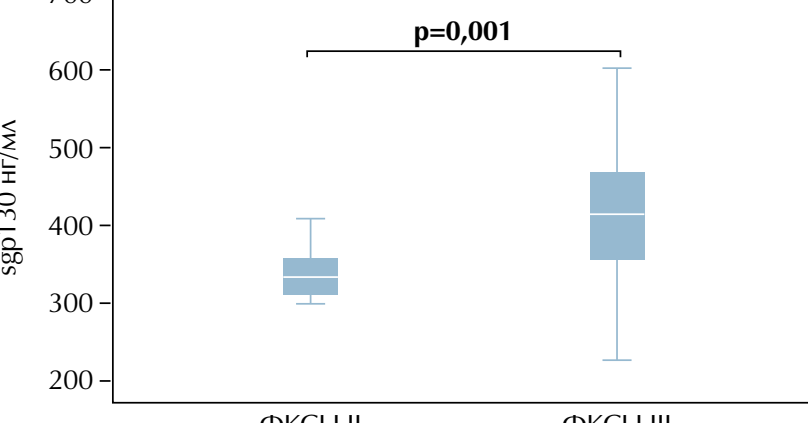

ФKCH II

ФKCH II

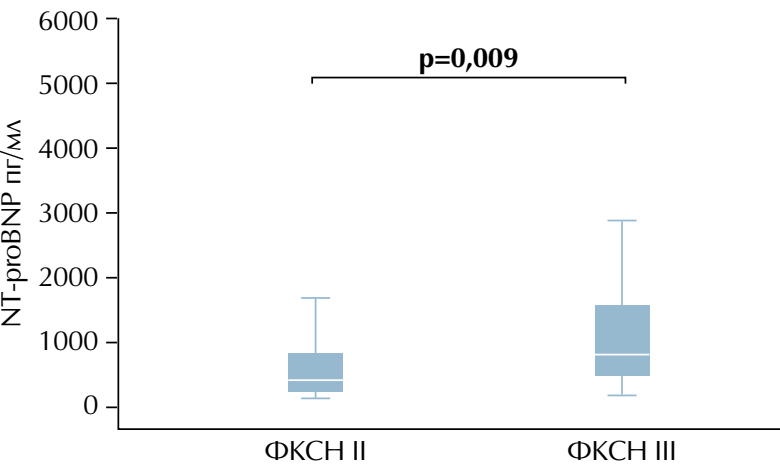

Максимальные и минимахьные значения, интерквартияьный размах имедианы концентрации sgp 130 и NT-proBNP; p - значение привецено мия критерия Манна - Уитни.

достаточности направлено на устранение тех факторов, которые привели к снижению насосной функции миокарда. Наиболее частыми причинами развития ХСН являются ИБС, АГ и ДКМП. При всех этих заболеваниях в крови пациентов отмечается изменение уровней провоспалительного ИЛ-6, и, следовательно, могут меняться уровни sgp130, влияющего на проведение сигнала ИЛ-6 по транссигнальному пути.

В настоящем исследовании в группах пациентов, сформированных в соответствии с этиологией ХСН (ИБС, АГ и ДКМП), достоверных различий между показателями sgp130 не обнаружено. Возможно, исследованные причины ХCH схожим образом влияют на уровни sgp 130 .

ХСН является прогрессирующим заболеванием. Для описания степени тяжести заболевания традиционно используют ФК. Мы оценили уровни sgp130 у пациентов II и III ФК. Циркулирующие уровни sgp130 повышалис при прогрессировании XCH.

Полученные нами данные согласуются с данными других исследователей. В частности, средние уровни sgp130 были выше у пациентов, у которых развилось ухулшение сердечной недостаточности, требуюшее госпитализации, внутривенной терапии или срочной рансплантании серл пациентами [14].

В исследовании Ritschel с соавт. показано, что повышенные уровни sgp130 в крови паниентов с ХCH
Рисунок 4. Корреяяиия межау уровнями sgp130 и NT-proBNP у пашиентов с XCH. R - коэффициент корреяяции Спирмена, р-значимость

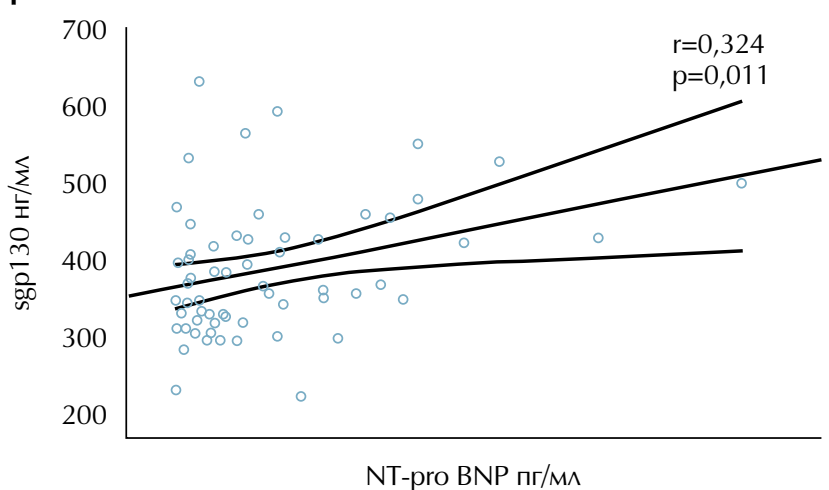

NT-pro BNP nr/MN

ассоциированы с сердечно-сосудистым повреждение и смертностью, вызванными ухудшением течения заболевания [15].

Многоцентровое исследование CORONA, анализирующее связь sgp130 с рисками сердечно-сосудистых событий у пожилых пациентов ( 260 лет) с ХСН ишемической природы, выявило ассоциацию повышенного уровня sgp 130 с различными причинами смертности, в том числе от сердечно-сосудистых событий [16]

Неблагоприятный прогноз у пациентов с $\mathrm{XCH}$ а также чувствительность пациентов К проводимой терапии оцениваются с помощью определения уровне NT-proBNP [17]. NT-proBNP продуцируется в миокарде желудочков сердца в ответ на растяжение и нагрузку давлением. Показано, что у пациентов с ХCH уровни sgp130 в перикардиальной жидкости положительно коррелировали с уровнями NT-proBNP [18]. В настоящем исследовании выявлена положительная корреляция между уровнями sgp130 и NT-proBNP в крови пациентов с XCH.

\section{ЗакАючение}

Таким образом, циркулирующие уровни sgp 130 повышаются при прогрессировании ХСН вне зависимосп этиологии заболевания. Установлена прямая зависимость между уровнями sgp130 и NT-proBNP. Рассмотрение sgp130 в качестве дополнительного показателя выраженности симптомов ХСН требует дальнейшего изучения.

\section{Авторы заявляют об отсутствии конфликта интересов.}

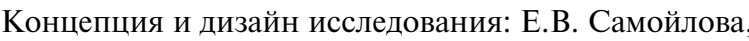
А. Коротаева, И.В. Жиров, С.Н. Терещенко Сбор и обработка материала: Т.В. Горю

Статистическая обработка: Е.В. Самойлова

Написание текста: Е.В. Самойлова, А.А. Коротаева,

Д.Р. Миндзаев, Д.А. Чепурнова

Редактирование: Е.В. Самойлова, А.А. Коротаева
ЛИTEPATYPA/REFERENCES

Allen L.A., Stevenson L.W., Grady K.L., Goldstein N.E., Matlock D.D. Arnold R.M., Cook N.R., Felker G.M., Francis G.S., Hauptman P.J, Havranek $\mathrm{E}$. American Heart Assosiation. Circulation. 2012; 125:1928-1952, https://doi org/1161/CIR.06013e31824242173

Richards A.M.M. Troughton R.W. Use of natriuretic peptides to guide and
monitor heart failure therapy. Clin Chem. 2012; 58:62-71, https//doi org/10.1373/clinchem. 2011.165704

Jug B., Salobir B.G., Vene N., Sebestjen M., Sabovic M., Keber I. Interleukin-6

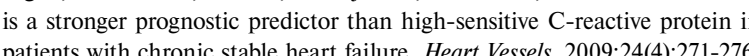
https://doi.org/ 10.1007/s00380-008-11111-4

Cainzos-Achirica M., Enjuanes C., Greenland P., McEvoy J.W., Cushman M., Dardari Z., Nasir K., Budoff M.J., Al-Mallah M.H., Yeboah J., Miedem of interleukin 6 in multiple chronic diseses and all-cuse deanth The Tulue Ethnic Study of Atherosclerosis (MESA). Atherossclerosisis 2018; 278:217-225.

httpps:///dio.org / 10.1016/j.atherosclerosisi.2018.09.034
Chow S.L., Maisel A.S., Anand I., Bozkurt B., Je Boer R.A., Felker G.M. T.J., Yancy C.W. Zile M.R. Role of biomarkers forthe preven, Liu P.P., Wang and management of heart failure: a scientific statement from the America heart association. Circulation. 2017; 135: e1054-e1091, https://doi.org/ 10.1161/
CIR.000000000000490 Lyngbakken M.N., Myhre P.L., Rosio H., Omland T. Novel biomarkers of
cardiovascular disease: Applications in clininaca practice. Crit Rev Clin Lab Sci. 2019:56(1):33-60, https://doi.org//10.1080/10408363.2018.1525335

7. Morieri M.L., Passaro A., Zuliani G. Interleukin-6 (Trans-Signaling) and Ischemic Vascular Disease: The Important Role of Soluble gpp 30.
Inflamm. 2017; 2017:1396398, https://dii.org/ 10.1155/2017/1396398

Jostock T., M llberg J., Ozbek S., Atreya R., Blinn G., Voltz N., Fischer soluble interleukin-6 6 recentor transsignaling responses $E u$. $J$. B B ich 2001;268(1):160-167, https://doi. org/1010.1046/ji.1432-1327.2001.01867

9. Чепурнова Д.А., Самойлова Е.В., Анисимов А.А., Верин А.Д., Коро-

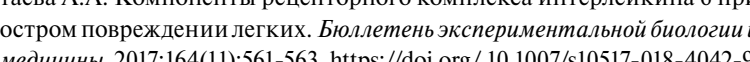

Inta I., Weber D., Grundt C., Veltkamp R., Winteroll S., Auffarth G.U serum concentrations with arterian blood pressure. J. Hypertens. 2009:27. 527-534, https://doi.org/ 10.1097/HJH.0b0133232831e9948

D.A., Zhitareva I.V., Shuvalow

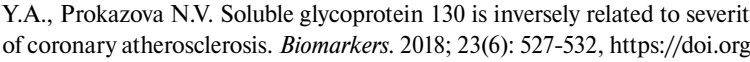
10.1080/1354750X.2018.1458151

I2. Ichiki T., Jougasaki M., Seloguchi M., Shimokawahara H., Nakashima H of soluble glycoprotein 130 in acute myocardial infarction. J. Cardiol. 2007 .
and 50(2):101-109, PMID:17802693.

Hirota H., Izumi M., Hamaguchi T., Sugiyama S., Murakami E., Kunisach K., Fujio Y., OShima Y., N heart failure. Heart Vessels. 2004; 19:237-241, https://doi.org/10.1007/s00380-

004-0770-z
M. Gwechenberger, R. Pacher, R. Berger et al. Comparison of soluble of heart failure progression. Journal of Heart and Lung Traneslantation 2005;24(12): 2190-2195, https://doi.org/ 10.1016/j.healun.2004.10.015

5. Ritschel V.N., Seljeflot I., Arnesen H., Halvorsen S., Weiss T., Eritslan J. and Andersen G.Q. IL6 signalling in patients with acute STelevation
myocardial infarction. Results Immunol. 2013; $4: 813$, https://doi.org/10.1016 rinim.2013.11.002

16. Askevold E.T., Nymo S., Ueland T., Gravning J., Wergeland R., Kjekshu Soluble gly A., Cleland J.G., McMurray J.J., Aukrust P., Gullestad L. analysis from the Controlled Rosuvastatin Multinational Trial in Heart Failure (CORONA). Circ Heart Fail. 2013;6(1):91-98, https://doi.org/10/1161/

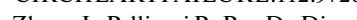

Dierckx R., Clark A.L., Cleland J.G.F. Dynamic risk stratificication using serial measurements of plasma concentrations of 269:196-200, https://doi.org/10.1016/j/.ijcard.2018.06.07

18. Iskandar R., Liu S., Xiang F., Chen W., Li L., Qin W., Huang F., Chen X. Expression of pericardial fluid Teells and related inflammatory cytokines in
patients with chronic heart failure. Exp. Ther. Med. $2017 ; 135(5): 1850-1855$ patients with chronic heart falure.
https://dio.org/10.3892/etm.2017.4202

Поступила 18.03.2019 Принята в печать 24.07.2019 